\title{
The Embodied Morphemes of Gaze
}

\author{
Isabella Poggi ${ }^{\circ}$ and Francesca D’Errico ${ }^{\circ \circ}$ \\ ○ Dipartimento di Scienze dell’Educazione \\ Università Roma Tre \\ Via Manin 53 - 00151 Roma - Italy \\ poggi@uniroma3.it \\ ${ }^{\circ}$ Facoltà di Scienze della Comunicazione \\ Università Sapienza di Roma \\ Via Salaria 113 - 00198 Roma - Italy \\ francesca.derrico@uniroma1.it
}

\begin{abstract}
The paper presents some studies aimed at singling out the meanings of specific items of gaze, while argueing that the values on some parameters of gaze items are not comparable to phonemes in a verbal language, but rather to morphemes, since by themselves they convey specific meanings.
\end{abstract}

Keywords: Communication, Gaze, Mimics, Morphemes.

\section{Gaze as a communicative system}

Gaze communication has captured attention in theory, empirical research and Embodied Agents. Gaze is studied in face-to-face interaction, imagery and text comprehension, persuasion, interest, and its semantic subtleties are simulated in Embodied Agents. Most studies, though, generally investigate a single dimension of eye gaze: gaze direction. Where people look at is very meaningful, to ask and to give feedback, to show attention or concentration. But many other aspects of eye behavior are relevant, and the function of gaze is not only to establish or maintain the setting for interaction, but to tell things: gaze conveys specific meanings, and makes up a rich and consistent communicative system to be described in a systematic way [1].

As for any communicative system, in gaze you can distinguish the signal - the morphological features and muscular actions displayed in the area of eyes - and the meaning - imagistic or conceptual representation linked to that signal. On the signal side, to describe the "phonology" of gaze ("optology", [1]), a number of parameters were singled out whose combination gives all the possible items of gaze: eyebrow movements, eyelid position, tension and movement, humidity and reddening of eyes, pupil dilation, focusing, direction of the iris with respect to direction of the Speaker's head and of the Interlocutor, duration of movements.

On the meaning side, gaze conveys various types of information [1]: entities (e.g., the so-called deictic gaze, eyes pointing to something, means "I refer to that thing there”, where the entity must be specified through contextual information), properties (squeezed eyes = small, subtle), performative (fixed stare = "I defy you"), emotion (inner parts of eyebrows up = I am sad), certainty (small frown = I'm am serious, not kidding), metacognitive (eyes left-downward = I'm trying to remember), topic- 
comment (eyebrows raised $=$ emphasis), turn-taking (open eyes wide = I want to speak) feedback (frown = I don't understand).

\section{Gaze morphemes}

To state the meaning of specific gaze items two studies were conducted [2] having an Embodied Agent not as an end but as a research tool. The first study investigated whether people attribute meanings to specific gaze items in a systematic and shared way. By using Greta's “face-library” [3], a tool to set a face in whatever facial expression by changing facial parameters of head, mouth and eye region, the values in three parameters were varied: eyelids aperture, eye direction and eyebrows position; by combining them 10 items of gaze were constructed, and for each the meaning was hypothesized (e.g., middle eyelids aperture, eyes directed to interlocutor, internal parts eyebrows down = I hate you). A questionnaire of multiple choice and open questions about the 10 items was submitted to 100 subjects. It resulted that the meanings hypothesized are fairly shared, and even the meanings proposed in open questions share some semantic components with those hypothesized.

A second aimed to assess if specific values in the parameter of eyelid aperture have an "optological" relevance, i.e., if they distinguish minimal pairs of gaze items. A questionnaire of 15 items was submitted to 100 subjects. The results showed that wide open, default, and half-closed eyelids distinguish minimal pairs of gaze items. However, here a specific value is not simply distinctive of a meaning against another, like for words, where phonemes are not meaningful per se; a value in gaze parameters looks meaningful in itself. For example, a component of "relaxation" is present in all the gaze items that contain the value the "half-closed eyelids". Relaxing means lowering the level of one's physical or mental energy; and you may do so either because energy leaves you (like in the meanings "I am about to faint”, "tired”, "going to sleep", "I am stupid”), or because you do not need much energy, or you feel justified not to exploit it, ("bored”, “extranged”, “Yes”, "I’ll pass over it”); or finally because you confront a too difficult goal which is not worth to waste energy ("I am resigned”, “Oh no, please!”).

But if a single value in a parameter bears meaning by itself, it has not simply a phonemic, but a morphemic import: it is an "embodied gaze morpheme", having its roots in the physiological reactions driven by, or linked to, specific affective or cognitive states. Thus, further morphemes of gaze will be investigated in subsequent research.

\section{References}

1. Poggi, I.: Mind, hands, face and body. A goal and belief view of multimodal communication. Weidler, Berlin (2007).

2. Poggi, I., Roberto, E.: Meaningful eyes. In: E.Ahlsén, P.J.Henrichsen, R.Hirsch, J.Nivre, A.Abelin, S.Stroemqvist, S.Nicholson, \& B.Dorriots (Eds.), Communication - Action Meaning. A festschrift to Jens Allwood. Department of Linguistics, Goteborg University, Goteborg, pp. 325-341 (2007).

3. Bevacqua, E., Mancini, M., Niewiadomski,R., Pelachaud, C.: An expressive ECA showing complex emotions. In: Language, Speech and Gesture for Expressive Characters, AISB 2007, Newcastle,UK, (2007). 